\title{
FIRST OPERATING EXPERIENCE WITH THE CERN DECELERATING RFQ FOR ANTIPROTONS
}

\author{
A.M. Lombardi, W. Pirkl, CERN, Geneva, Switzerland \\ Y. Bylinsky, Institute for Nuclear Research [INR], Moscow
}

\section{Abstract}

The RFQD decelerates antiprotons from a momentum of $100 \mathrm{MeV} / \mathrm{c}$ (kinetic energy $5.31 \mathrm{MeV}$ ) down to a kinetic energy variable between $\sim 10 \mathrm{keV}$ and $120 \mathrm{keV}$. A novel feature is the implementation of a floating internal $\mathrm{RF}$ structure, mounted on $\mathrm{HV}$ insulators, to allow continuous post-deceleration or acceleration by a DC bias. A description of the system is given, followed by reports on the first operating experience with the ASACUSA experiment, dedicated performance measurements and consolidation progress.

\section{DESCRIPTION OF THE SYSTEM}

The RFQ decelerates the beam ejected from the Antiproton Decelerator ring AD [1] at CERN. The key parameters are given in table 1 below.

Table 1: RFQ key parameters

\begin{tabular}{|l|l|}
\hline Operating frequency & $202.5 \mathrm{MHz}$ \\
\hline Shunt impedance & $13.8 \mathrm{k} \Omega$ \\
\hline Vane voltage & $167 \mathrm{kV}$ \\
\hline Maximum electric field & $33 \mathrm{MV} / \mathrm{m}$ \\
\hline Dissipated power & $1.1 \mathrm{MW}$ \\
\hline Minimum aperture radius & $0.4 \mathrm{~cm}$ \\
\hline Max. vane modulation factor & 2.9 \\
\hline Input energy & $5.314 \mathrm{MeV}$ \\
\hline Output energy with internal & $63 \mathrm{keV}(\mathrm{RFQ})$ \\
DC post-deceleration & $\pm 60 \mathrm{keV}(\mathrm{DC})$ \\
\hline Transverse acceptance & $15 \pi \mathrm{mm} \mathrm{mrad}$ \\
\hline Phase/energy acceptance & $\pm 10^{\circ} \pm 0.9 \times 10^{-3}$ \\
\hline Decelerating efficiency (theory) & $45 \%$ \\
\hline Number of particles at input & $2.4 \cdot \times 10^{7}$ \\
\hline Nominal pulse rate (test) & $1 / 2.4 \mathrm{~s}^{-1}$ \\
\hline
\end{tabular}

\subsection{Basic system concept}

Since the output energy of an RFQ is essentially fixed, some other kind of variable post-acceleration or deceleration has to be provided. Variation by RF (e.g. by a cavity) cannot provide sufficient range due to the limited flat-top of a single sinusoid relative to the large beam length at the output. Constant energy variation over the full RF cycle can be provided by a DC bias. However, mounting the physics apparatus or the RFQ on a floating $\mathrm{HV}$ platform is excluded for practical reasons. A novel solution is to mount the internal RF structure of the RFQ on insulators to apply the energy correcting DC voltage (see figure 1). The bias on the structure acts not only on the output energy but also shifts the input energy in the opposite direction. To compensate for this unwanted effect, an energy corrector cavity is provided directly at the input flange of the RFQ.

In a decelerating RFQ, smooth bunching of the beam by the same approach as in accelerating RFQs leads to prohibitive overall length [2]. External bunching had therefore to be foreseen using a separate buncher cavity located $6.15 \mathrm{~m}$ upstream of the RFQ. This limits the overall decelerating efficiency to less than $50 \%$. The RFQ acts, nevertheless, as an efficient beam transport also for particles outside the longitudinal acceptance. Therefore virtually the full beam intensity within the transverse acceptance appears at the output, albeit with only partial or no deceleration.

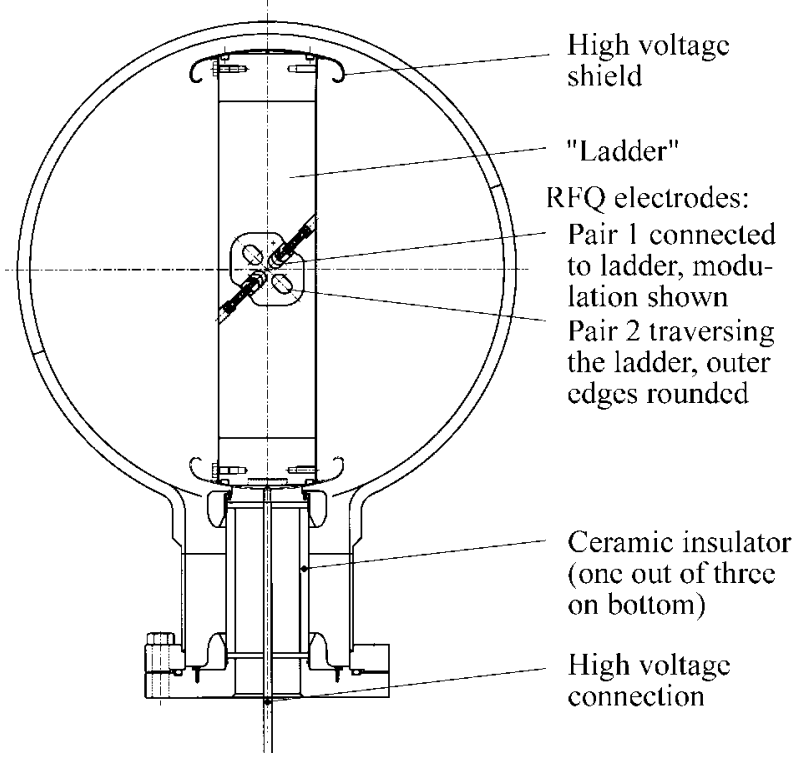

Figure 1: Cross section of the RFQ

\subsection{Short description of the hardware}

A complete description of the system is found in [3], of which the main points are repeated in the following.

The RFQ is of the 4-rod type, where the "rods" are rectangular electrodes mounted at $\pm 45^{\circ}$ in a symmetric, ladder-like RF resonating and supporting structure. SPIRASHIELD ${ }^{\text {TM }}$ RF contacts of $1.2 \mathrm{~mm}$ diameter are provided at each connection interface. The ladder is composed of 34 RF cells with a total length of $3415 \mathrm{~mm}$, held in place by 5 vertical ceramic insulators, 2 on top and 3 on the bottom. The assembly is housed in a cylindrical tank made of stainless steel, copper plated on the inside to reduce the RF losses. 


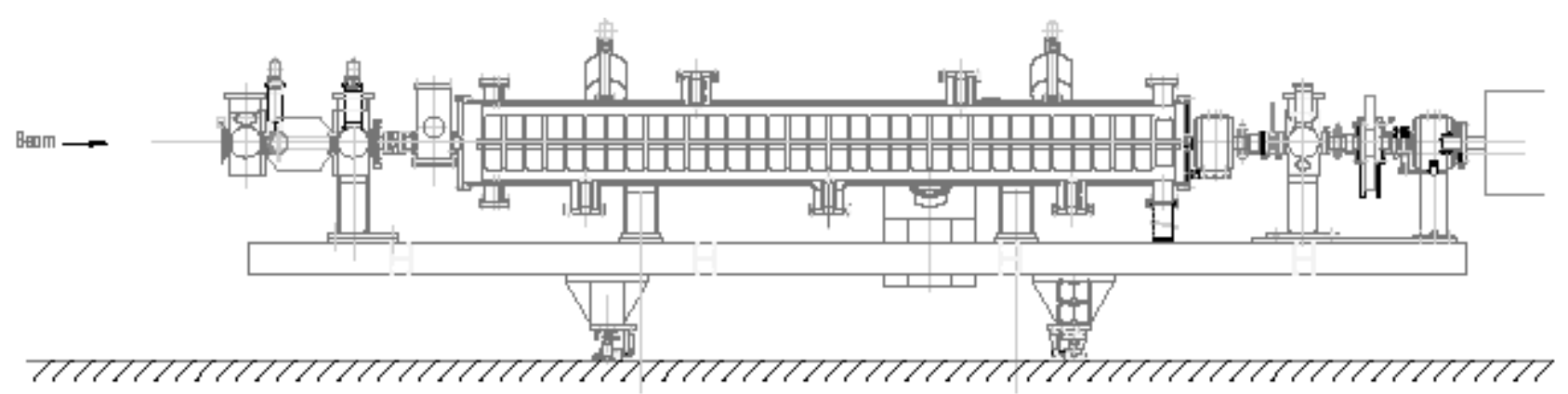

Figure 2: RFQ girder with MEBT (left), longitudinal section of the RFQ (mid), LEBT (right).

An aluminium girder of $6 \mathrm{~m}$ length carries the last part of the medium-energy beam transport "MEBT" (two diagnostic boxes and the energy corrector cavity), the RFQ tank and the low-energy beam transport "LEBT" (two solenoids, two steerers and a SEM monitor), see figure 2 .

\subsection{Beam Diagnostics}

The beam at the input of the RFQ is monitored by:

-the "watchdog": two perforated caesium-iodide scintillator screens, $1 \mathrm{~mm}$ thick, $543 \mathrm{~mm}$ apart, with holes of $24 \mathrm{~mm}$ and $17 \mathrm{~mm}$ respectively, which allow a $15 \pi$ $\mathrm{mm}$ mrad beam of the nominal divergence to pass unperturbed. The signals of beam tails outside this limit are captured by two synchronised cameras and frame grabbers:

-two full scintillator screens that can be moved over the watchdog screens, thus providing (destructively) information on the transverse beam parameters,

- a beam transformer.

One secondary emission (SEM) monitor called "HORIbox" is permanently mounted in the LEBT. It is $95 \%$ transparent and consists of a horizontal and a vertical grid of 32 gold-plated tungsten wires, $20 \mu \mathrm{m}$ diameter, read out by charge-sensitive preamplifiers [4]. A second HORI-box is provided either at the interface to the physics experiment or at the end of a dedicated measurement line.

Additional devices such as a Faraday cup or a silicon strip detector can be mounted in place of blind flanges that are provided in the measurement boxes or pumping ports in the beam lines.

\section{BEAM TESTS}

\subsection{Tests with Protons at Aarhus University}

The use of a proton beam is very attractive for the running in of the decelerating RFQ (fast repetition rate, high intensity, absence of annihilation, thus simplifying the diagnostics). Since no suitable proton beam was available at CERN, it was decided to follow an invitation to perform the initial tests at the Tandem accelerator of the University of Aarhus/Denmark.

A dedicated measurement line consisting of a spectrometer magnet, two steerers and a quadrupole triplet had been constructed. The diagnostics consisted of a measurement box for a Faraday cup together with a "HORI-box".

Two weeks of beam time were allocated for the RFQ and associated systems to be run-in. Procedures for the proper adjustment of RF settings were devised and recipes for systematic exploration of the parameter space were tested. Energy variation up to $120 \mathrm{keV}$ was demonstrated by a bias of positive polarity on the ladder.

Owing to the deceleration, the physical dimensions of the input beam are increased by about a factor of 3 at the $63 \mathrm{keV}$ output; the same factor applies for output/input variations. In the absence of "single-shot" instrumentation the reliability of the measurement results depends critically on the stability of subsequent pulses: transmission measurements compare different shots in the (destructive) Faraday cups, emittance reconstruction relies on a series of data during a quadrupole scan. Instabilities of the beam and multipactoring in the buncher prevented fine tuning and precise performance measurements. A low-energy antiproton content of about $25 \%$ could nevertheless be demonstrated at the output of the RFQ (ratio of the Faraday-cup readings at the end of the measurement line and in the LEBT, respectively). Also a vertical transverse emittance of about $60 \pi \mathrm{mm}$ mrad at 63 $\mathrm{keV}$ was measured, although for uncertain input beam conditions.

\subsection{Antiproton Tests at CERN}

Following the positive outcome of the proton tests, the RFQ was installed in the ASACUSA experimental area of the $\mathrm{AD}$ to provide the experiment ESA [5] with antiprotons. Useful beam was delivered quickly, thanks to the experience previously gained with the protons. The testline with the spectrometer magnet was initially kept between the RFQ and ESA to allow further assessment of the system performance. A low-energy antiproton content of at least $37 \%$ was measured at the output of the RFQ (see figure 3).

Considerable pulse-to-pulse variations caused by instabilities of the input beam were observed, further amplified by the cascading of two sensitive spectrometers in the measurement line and in the physics apparatus. The test line was therefore dismantled and the experiment connected directly at the end of the LEBT. This resulted in a very noticeable stability improvement, and "publishable" results could be obtained by ESA. 


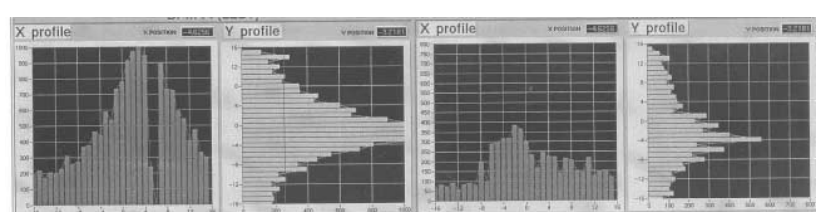

Figure 3: Display of two HORI-boxes with antiprotons behind RFQ (left) and end of test line (right).

The test period was interrupted by the 4-months wintershutdown of the CERN machines. Several improvements of the system were implemented, e.g. modification of the timing system and installation of a Faraday cage around the RF power amplifiers to eliminate any risk of RF interference.

In the buncher and energy-corrector cavities, multipactoring prevented stable operation in certain amplitude ranges. Continued conditioning cured this problem, although strong electron emission still persists which interferes with the nearby beam diagnostics. Improved vacuum conditions were prepared as ad-hoc prevention of this effect.

In the RFQ, positive bias on the ladder could be applied without restriction, but negative polarity led to excessive DC current and disruption of the power supply at nominal $\mathrm{RF}$ level. This was identified as multipactoring in accordance with theory predicting significantly wider multipactoring bands for negative DC bias combined with RF [6]. The HV supply was modified to cope with high pulse currents. Peak currents of up to $5 \mathrm{~A}$ were initially drawn by the multipactoring at $-40 \mathrm{kV} \mathrm{DC}$, but conditioning during one night reduced this by a factor of six. The remaining DC bias variation is negligible during the beam duration of a fraction of a microsecond, thus operation is assured over the full range of $\pm 60 \mathrm{kV}$ ladder bias.

After the shutdown the measurement line was reinstalled for a short dedicated RFQ test period. In addition to the HORI-boxes a Lucite Cherenkov counter was a helpful diagnostic tool to compare intensities of the beam that was deliberately stopped at different points in the installation. It was shown that the two modes of $A D$ ejection, with short or long bunch (respectively with high or low energy spread), had virtually no effect on RFQ transmission.

A first attempt was undertaken to measure the integrated number of low-energy particles at the end of the spectrometer line via ${ }^{24} \mathrm{Na}$ production by activation of a thin $\mathrm{Al}$ foil [4],[7]. Integrated over $>1$ hour an average overall transmission of about $20 \%$ was found as the ratio between the number of particles at the end of the spectrometer line compared to the number of particles in the $\mathrm{AD}$ ring just before ejection at $100 \mathrm{MeV} / \mathrm{c}$. The ratio fluctuated from pulse to pulse due to variations of the input beam and attained $25 \%$ according to the real-time reading of the Cherenkov counter.

The unexpectedly low transmission can be caused by losses in the spectrometer line (only the lower part of the aluminium foil had been found activated) and losses/mismatches in the $\mathrm{AD}$ ejection line (the beam transformer readings showed a substantially lower intensity at the RFQ input than expected).

From the end of the test period till the end of May 2001 the ESA experiment regularly took beam at RFQ output energies down to $15 \mathrm{keV}$. Fully satisfactory results were reported [8] despite of considerable fluctuations of the input beam. The RMS energy spread measured in the ESA electrostatic analyser was $5.7 \pm 0.5 \mathrm{keV}$ at $61.7 \mathrm{keV}$.

\section{FUTURE PLANS AND CONCLUSION}

Work remains to be done to reduce the electron emission in the small cavities and to improve the input diagnostics. Also, precise transmission data are not yet available, and should be measured together with other parameters as soon as the operational schedule permits. A new challenge for the RFQ is the imminent installation of a superconducting solenoid in the physics experiment. The resulting strong stray field in the LEBT, together with different requirements for spot size and beam length, may necessitate additional studies and/or hardware modifications.

However the validity of the decelerating RFQ configuration with DC output energy variation has been demonstrated. The possibility to compensate for energy variations of the input beam by the energy corrector cavity at the input of the RFQ has emerged as an additional bonus of the scheme. It can be considered as a very promising tool for low-energy antiproton physics.

\section{ACKNOWLEDGEMENTS}

The realisation of the project would not have been possible without the support of the ASACUSA Collaboration and the help of many different groups at CERN. The hospitality of the Aarhus group during the beam tests was exceptional and memorable. Special thanks go to M. Hori for his contribution of the HORIboxes and continued help and interest.

\section{REFERENCES}

[1] S. Maury et al., "Commissioning and First Operation of the Antiproton Decelerator (AD)", this conference.

[2] A.M. Lombardi, "Accelerating and Decelerating RFQs," Proc. Linear Accelerator Conference Chicago 1998, 377-381.

[3] Y.Bylinsky, A.M. Lombardi, W.Pirkl, "RFQD - A decelerating Radio-Frequency Quadrupole for the CERN Antiproton Facility" Proc. Linac 2000 Conference, Monterey, 554-556.

[4] M.HORI, private communication (paper in preparation).

[5]H.Knudsen et al, "Construction of an Electrostatic Analyzer (ESA) for Energy Loss Measurements in the CERN AD", CERN PS/CA Note 99-23.

[6] P. Ylä-Oijala "Suppressing Electron Multipacting in Coaxial Lines," TESLA 97-21, November 1997.

[7] P.Lubinski et al, Phys. Rev. Lett. 73, 3199 (1994).

[8] H. Knudsen, private communication (paper in preparation) 